Bundesgesundheitsbl $2021 \cdot 64: 426-434$ https://doi.org/10.1007/s00103-021-03301-4 Eingegangen: 17. November 2020

Angenommen: 25. Februar 2021

Online publiziert: 21. März 2021

c c Der/die Autor(en) 2021

Ruben Rose ${ }^{1,2} \cdot$ Damian Scherer ${ }^{1,2} \cdot$ Gregor Maschkowitz $^{1,2} \cdot$ Christoph Läubrich $^{1,2}$. Helmut Fickenscher ${ }^{1,2}$

${ }^{1}$ Kompetenzzentrum für das Meldewesen übertragbarer Krankheiten in Schleswig-Holstein, am Institut für Infektionsmedizin, Christian-Albrechts-Universität zu Kiel und Universitätsklinikum SchleswigHolstein, Kiel, Deutschland

${ }^{2}$ Institut für Infektionsmedizin, Christian-Albrechts-Universität zu Kiel und Universitätsklinikum Schleswig-Holstein, Kiel, Deutschland

\title{
COVID-19 in Schleswig-Holstein: Infektionsepidemiologische Auswertungen von März bis September 2020
}

Meldung der Erkrankung sind als Kriterien definiert: das klinische Bild der Pneumonie, ein unspezifisches Bild mit akuter respiratorischer Symptomatik jeder Schwere oder der krankheitsbedingte Tod. In dieser Arbeit werden nur Fälle mit Erregernachweis und somit Erfüllung der Referenzdefinition nach dem Stand des 29.05.2020 untersucht.

Die namentliche Meldepflicht basiert auf $\$ 6$ Abs. 1 Satz 1 Nr. 1 Buchst. t IfSG und schließt den Krankheitsverdacht, die Erkrankung sowie den Tod ein. Nach $₫ 7$ Abs. 1 Nr. 44a IfSG umfasst die namentliche Meldepflicht den direkten Nachweis von SARS-CoV-2, soweit er auf eine akute Infektion hinweist. $\mathrm{Zu}$ Beginn der Pandemie wurden Nachweise von SARS-CoV-2 der allgemeinen Meldekategorie „Weitere bedrohliche Krankheiten" (WBK) zugeordnet. Ab dem 17.04.2020 wurden sie im zentralen Meldeprogramm SurvNet der neuen Kategorie CVD (für COVID19) zugewiesen. Eine kurze Übersicht folgt über die wichtigsten Ereignisse im Zusammenhang mit der COVID19-Pandemie mit Fokus auf SchleswigHolstein. Bereits am 01.12.2019 wurden erste COVID-19-Fälle in Wuhan in China beobachtet. Am 01.01.2020 wurde ein Markt für Fisch und Schlachttiere in Wuhan geschlossen. Am 13.01.2020 wurde der erste Erkrankungsfall außerhalb Chinas in Thailand berichtet. Die
Abriegelung der Stadt Wuhan erfolgte am 23.01.2020. Die Weltgesundheitsorganisation (WHO) erklärte am 30.01.2020 die ,gesundheitliche Notlage internationaler Tragweite" und am 11.03.2020 den Pandemiefall. Nachdem am 27.02.2020 der erste Erkrankungsfall in Deutschland in der Region München gemeldet wurde, fand am 28.02.2020 die Meldung des ersten Erkrankungsfalls für Schleswig-Holstein statt. Der erste Todesfall eines Schleswig-Holsteiners in Ägypten wurde am 08.03.2020 berichtet und der erste Todesfall in Schleswig-Holstein am 17.03.2020. Am 18.03.2020 trat ein einschränkendes Maßnahmenpaket in Schleswig-Holstein in Kraft, das unter anderem die Schließung der Schulen und Kindergärten sowie der Restaurants, Besuchsverbote in Kliniken und die Untersagung öffentlicher Veranstaltungen einschloss. Über weitere Landesverordnungen folgten das Tourismusverbot in Schleswig-Holstein und wesentliche Kontaktbeschränkungen. Am 25.03.2020 erklärte der Bundestag die „epidemische Lage von nationaler Tragweite" und verabschiedete am 27.03.2020 das erste "Gesetz zum Schutz der Bevölkerung bei epidemischer Lage von nationaler Tragweite" sowie am 19.05.2020 das zweite Gesetz mit gleicher Bezeichnung. Seit dem 05.05.2020 wurden schrittweise Lockerungen der einschränkenden Maßnahmen wirksam. Die Landesver- 


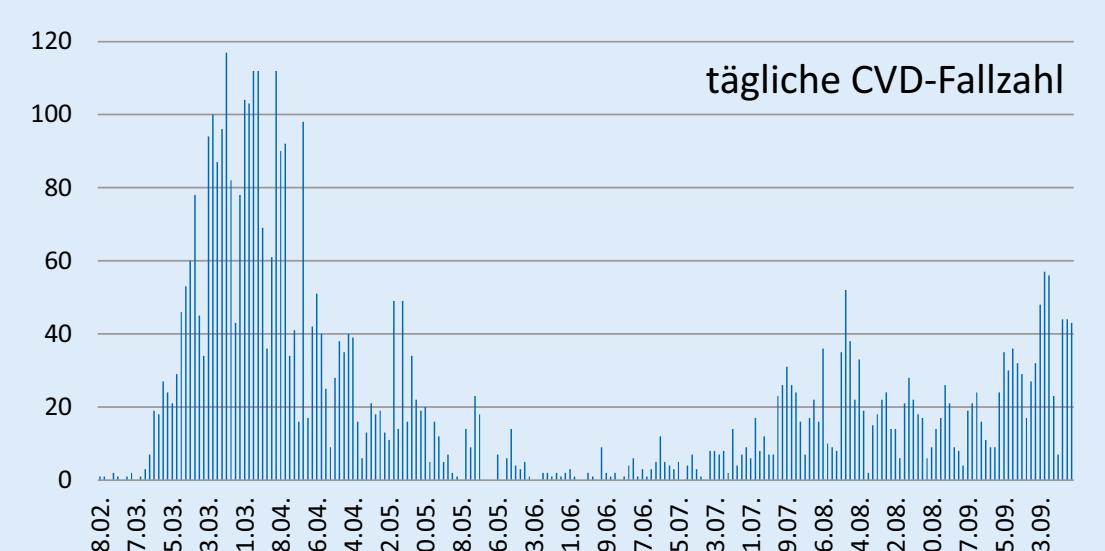

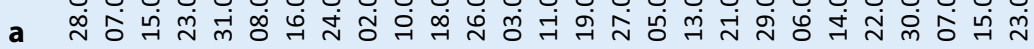

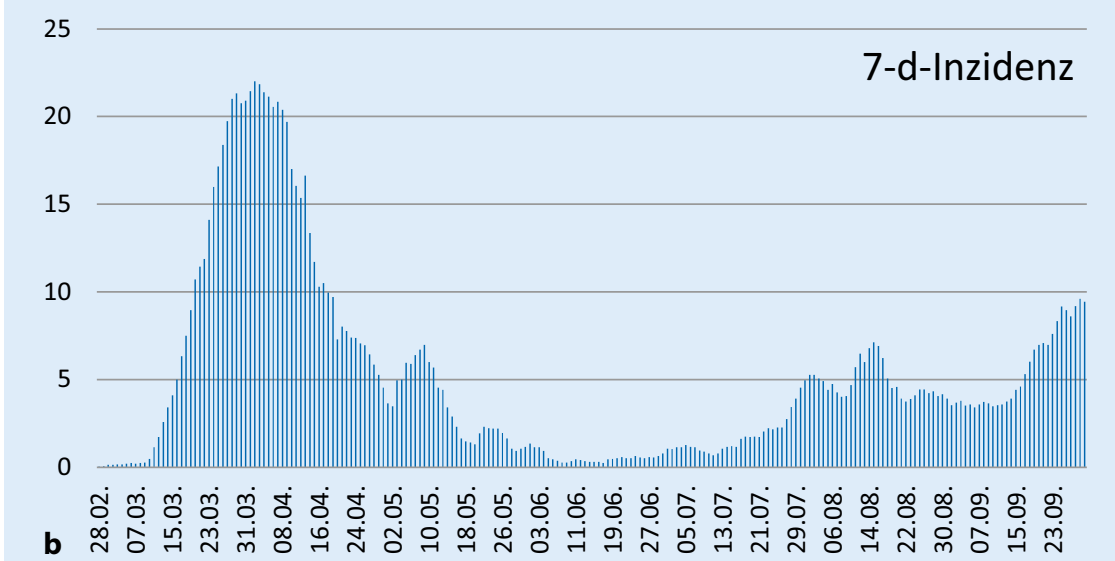

Abb. 1 \ Tägliche Anzahl der Meldungen (a) und 7-Tage-Inzidenz (b) in Schleswig-Holstein im Zeitraum März bis September 2020. Die erste epidemische Phase wurde ab der Meldung des ersten Falls Ende Februar bis Ende Mai definiert, die zweite Phase von Juni bis September 2020. Die 7-Tage-Inzidenz errechnet sich aus der Summe der Fälle über die zurückliegenden 7 Tage bezogen auf 100.000 Einwohner. CVD Meldekategorie COVID-19 im zentralen Meldeprogramm SurvNet des Robert KochInstituts

ordnungen Schleswig-Holstein wurden mehrfach aktualisiert.

In diesem Bericht mit Datenstand zum 09.10.2020 wird die Epidemiologie der SARS-CoV-2-Pandemie in Schleswig-Holstein im Zeitraum März bis September 2020 beschrieben. Bereits vorliegende Auswertungen der Landesmeldestelle Schleswig-Holsteins zu der ersten Pandemiewelle werden damit fortgeschrieben [2].

\section{Auswertung der Meldedaten von März bis September 2020}

Im Beobachtungszeitraum März bis September 2020 wurden in Schleswig-
Nach der Einführung einschränkender Maßnahmen wurde die Ausbreitung zeitnah verlangsamt und schon Anfang Juni (Beginn der zweiten Phase) lag nur noch eine minimale Fallzahl vor. Im Monat Juli wurde ein deutlicher Anstieg der täglichen Fallzahlen und der 7-Tage-Inzidenzen (pro 100.000 Einwohner) beobachtet, auf den dann in der zweiten Hälfte des Septembers ein weiterer Anstieg auf ca. die Hälfte der täglichen Fallzahlen und der 7-Tage-Inzidenzen der ersten Phase folgte ( $\mathbf{A} \mathbf{b} b . \mathbf{1} \mathbf{a}, \mathbf{b})$. Die zweite Phase setzte sich weit über den Jahreswechsel im Jahr 2021 fort, allerdings mit veränderten Schwerpunkten.

In der ersten Phase lag der Schwerpunkt der geografischen Verteilung in den Hamburg-nahen Kreisen Pinneberg und Stormarn; in der zweiten Phase wirkten sich unübersichtliche Ausbruchssituationen im Kreis Dithmarschen und in Neumünster zusätzlich stark aus (• Abb. 2a, b). Die Altersgruppenverteilung der ersten Phase war durch niedrige Fallzahlen bei Kindern und Jugendlichen sowie durch deutlich erhöhte Fallzahlen bei älteren Personen geprägt. Im Gegensatz dazu betraf die zweite Phase hauptsächlich junge Erwachsene und vergleichsweise in geringem $\mathrm{Maß}$ ältere Personen ( $\bullet$ Abb. 2c, d).

Der jüngste Patient war ein Säugling, der älteste Patient war 119 Jahre alt. Ab dem 45. Lebensjahr stieg die Hospitalisierungsrate kontinuierlich an und ab dem 60. Lebensjahr steigerte sich die Letalität. Von den 163 verstorbenen infizierten Patienten wurde die Todesursache bei 146 Fällen der SARS-CoV-2-Infektion zugeschrieben, bei 17 Fällen wurde eine andere Todesursache festgestellt und in einem Fall fehlte diese Angabe. Insgesamt 120 der Verstorbenen waren im Rahmen der Erkrankung hospitalisiert.

Die Kernparameter wurden bei asymptomatischen, symptomatischen und hospitalisierten SARS-CoV-2-infizierten Personen und Verstorbenen vergleichend betrachtet ( $\bullet$ Tab. 1 und 2). Hierfür wurden alle 4827 Personen mit PCR-Nachweis des SARS-CoV-2 und entsprechenden Angaben in vier Gruppen eingeteilt: 1. infizierte Personen ohne Symptomatik oder mit einer für COVID-19 nicht relevanten Sympto- 
matik; 2. symptomatische Personen mit relevanter Symptomatik, aber ohne hospitalisierte oder verstorbene Personen; 3. hospitalisierte Personen, aber ohne Verstorbene; 4. verstorbene Personen ungeachtet einer vorherigen Hospitalisierung.

Die Inzidenzen (jeweils bezogen auf 100.000 Einwohner) variierten in der ersten Phase von 34 (Ostholstein) bis 190 (Pinneberg) und in der zweiten Phase von 15 (Schleswig-Flensburg) bis 114 (Dithmarschen). Die Hospitalisierungsraten rangierten in der ersten Phase zwischen 4,8\% (Lübeck) und $22,9 \%$ (Kiel) und in der zweiten Phase zwischen 2,7\% (Flensburg) und 19,1\% (Plön). Bezüglich der Letalität lag in der ersten Phase das Maximum bei 7,9\% (Stormarn) und in der zweiten Phase bei 2,0\% in Nordfriesland, während aus Ostholstein in diesem Zeitraum kein Todesfall berichtet wurde (• Tab. 1).

Während die Geschlechterverteilung insgesamt ausgeglichen war $(n=2336$ männlich, $n=2491$ weiblich), überwogen bei den Verstorbenen die Männer ( $n=97$; Letalität $4,2 \%)$ im Vergleich zu den Frauen $(n=65$; Letalität $2,6 \%$; - Tab. 2). Das durchschnittliche Alter aller infizierten Personen betrug in der ersten Phase 50,7 Jahre und in der zweiten Phase 34,3 Jahre ( $p=0,0047$ im ungepaarten t-Test). Ebenfalls waren die Altersunterschiede hospitalisierter Personen im Vergleich der beiden Phasen hoch signifikant $(p=0,0014$ im ungepaarten $t$-Test bei einem Mittelwert von 68,4 Jahren in der 1. Phase und 55,9 Jahren in der 2. Phase). Das durchschnittliche Alter der Verstorbenen war 79,2 Jahre und lag bei Frauen mit 83,2 höher als bei Männern mit 76,6 Jahren.

Die häufigsten Symptome waren in beiden Phasen Fieber (37,1 \% vs. 22,9\%), Husten $(47,7 \%$ vs. $27,5 \%)$ und Allgemeinsymptome $(41,1 \%$ vs. $34,6 \%)$. Veränderungen der Geruchs- und Geschmackssinne wurden in der ersten Phase noch nicht systematisch erfasst und sind daher unterschätzt, während sie in der zweiten Phase bei 13,2\% der infizierten Personen auftraten. Die häufigsten Risikokonstellationen für schwere Verläufe von SARS-CoV-2-Infektionen betrafen das Herz-Kreislauf-System

Bundesgesundheitsbl $2021 \cdot 64: 426-434$ https://doi.org/10.1007/s00103-021-03301-4 (c) Der/die Autor(en) 2021

R. Rose · D. Scherer · G. Maschkowitz - C. Läubrich · H. Fickenscher COVID-19 in Schleswig-Holstein: Infektionsepidemiologische
Auswertungen von März bis September 2020

\section{Zusammenfassung}

Die COVID-19-Pandemie stellt das deutsche Meldewesen im öffentlichen Infektionsschutz vor große Herausforderungen. Im Bundesland Schleswig-Holstein unterstützt die Landesmeldestelle die Gesundheitsämter durch tägliche und wöchentliche Auswertungen und hilft bei der Übermittlung der Meldedaten gemäß Infektionsschutzgesetz an das Robert Koch-Institut.

In dem vorliegenden Bericht der Landesmeldestelle Schleswig-Holstein werden die SARS-CoV-2-Meldedaten aus dem Zeitraum März bis September 2020 ausgewertet. In Orientierung an der Entwicklung der Infektionszahlen wurde der Zeitraum in zwei Phasen ähnlichen Umfangs eingeteilt: März bis Mai und Juni bis September. Insgesamt wurden 4898 Infektionsfälle gemeldet. Bei dem Vergleich der Phasen zeigten sich besonders deutliche Unterschiede hinsichtlich der
Hospitalisierung und Letalität, des Alters und der Staaten des Infektionsorts. In der ersten Phase waren besonders ältere Personen von einer hohen Hospitalisierungsrate und Sterblichkeit betroffen. In der zweiten Phase lagen das durchschnittliche Alter und die Hospitalisierungs- und Sterberaten deutlich niedriger und ein besonders großer Anteil war mit internationaler Reiseaktivität verbunden. Die Auswertung der Ausbruchsdokumentationen ergab den besonderen Schwerpunkt im Setting der privaten Haushalte. Dieser Artikel beschreibt die epidemische Situation in einem im Bundesvergleich Niedriginzidenzland.

Schlüsselwörter Infektionen mit SARS-CoV-2 · COVID19. Schleswig-Holstein · Meldepflicht . Ausbruchsmeldungen

\section{COVID-19 in Schleswig-Holstein: infection epidemiological evaluations from March to September 2020}

\section{Abstract}

The COVID-19 pandemic poses major challenges for the German notification system in public infection control. For the federal state of Schleswig-Holstein evaluations, the state reporting office supports the public health departments by providing daily and weekly evaluations and supports the transmission of notification data to the Robert Koch Institute according to the Infection Protection Act.

In the present report of the state notification office of Schleswig-Holstein, the SARS-CoV-2 reporting data for the period from March to September 2020 are evaluated. Based on the development of the infection numbers, this period was divided into two phases of similar size: March to May and June to September. A total of 4898 infection cases were reported. Upon comparison of the phases, there were particularly marked differences in hospitalization and mortality, age, and countries of infection site. In the first phase, elderly persons were particularly affected by high rates of hospitalization and mortality. In the second phase, the average age and hospitalization and mortality rates were significantly lower, and a particularly large proportion were associated with international travel activity. The evaluation of the outbreak documentation revealed a particular focus in private household settings. This article describes the epidemic situation in a lowincidence state within the Federal Republic of Germany.

Keywords

SARS-CoV-2 infections · COVID-19 · SchleswigHolstein - Reporting of notifiable diseases . Outbreak reporting $(n=565)$, das Nervensystem $(n=205)$ und die Lungen $(n=205)$. Während der ersten Phase waren 17,2\% aller infizierten Personen asymptomatisch, während der zweiten Phase 38,7\%.
In den Meldedaten werden Fälle auch nach der Zugehörigkeit in Betreuung oder Tätigkeit unterschieden nach $\$ 23$ IfSG vor allem in Krankenhäusern und anderen medizinischen Funktionen, nach $\$ 33$ vor allem in Kitas, Kinderhor- 

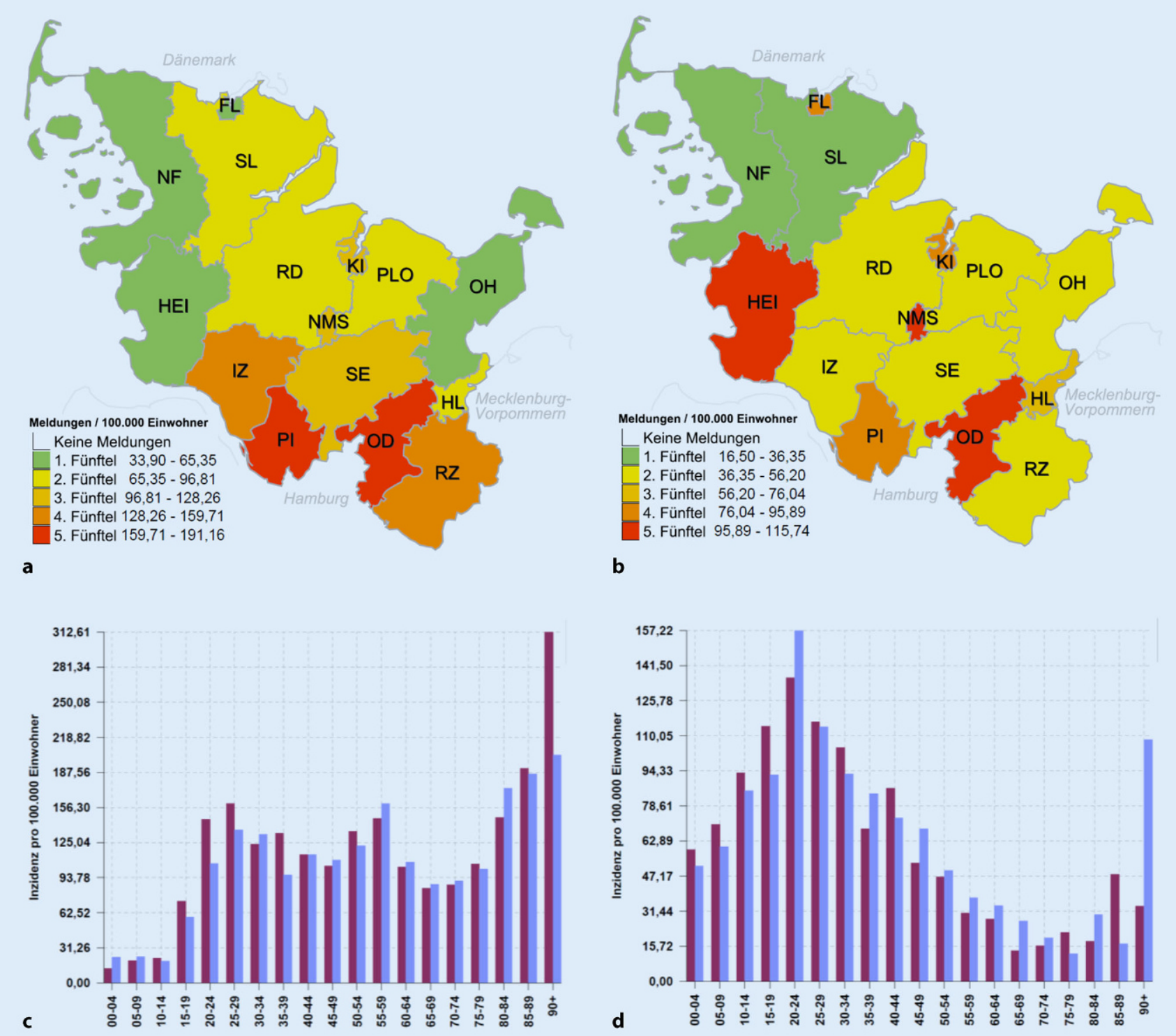

Abb. $2 \Delta$ Inzidenzen von SARS-CoV-2 in Schleswig-Holstein in den 2 epidemischen Phasen (links: Phase 1, rechts: Phase 2). a,b geografische Verteilung; c,d Alters- und Geschlechterverteilung, Darstellung nach Altersgruppen. Geschlecht weiblich (violett) oder männlich (blau). Phase 1: März bis Mai 2020; Phase 2: Juni bis September 2020. Die Erstellung der grafischen Auswertungen erfolgte mit dem Programmpaket IBIS (Läubi-Soft). FL Flensburg, HEI Dithmarschen, HL Lübeck, IZSteinburg, KI Kiel, NF Nordfriesland, NMS Neumünster, OD Stormarn, OH Ostholstein, PI Pinneberg, PLO Plön, RD Rendsburg-Eckernförde, $R Z$ Herzogtum Lauenburg, SE Segeberg, SL Schleswig-Flensburg

ten, Schulen, Heimen und Ferienlagern, nach $\$ 36$ vor allem in Pflegeeinrichtungen, Obdachlosenunterkünften, Einrichtungen für Asylsuchende, sonstigen Massenunterkünften, Justizvollzugsanstalten und nach $\$ 42$ vor allem in der Fleischindustrie und Küchen von Gaststätten und Gemeinschaftsverpflegung. Nach den $\$ \$ 23,33$ bzw. 36 des IfSG betreute Personen waren in insgesamt 107, 250 bzw. 340 Fällen infiziert, von denen 14, 0 bzw. 70 Personen verstar- ben (-Tab. 2). In den Bereichen nach $\$ \$ 23,33,36$ und 42 tätige Personen waren in 424, 101, 207 bzw. 215 Fällen infiziert. Bezüglich der ausländischen Expositionsorte dominierten in der ersten Phase Österreich und Südeuropa und in der zweiten Phase Südosteuropa und die Türkei. Nur wenige Personen mit ausländischem Expositionsort verstarben an den Folgen der Infektion $(n=8)$.

Im Beobachtungszeitraum wurden 412 Infektionsherde festgestellt, von denen 204 mindestens drei Personen umfassten. In der ersten Phase wurden 250 Herde mit insgesamt 1004 Personen dokumentiert, in der zweiten Phase 162 Herde mit 579 Beteiligten (• Tab. 3A). Bei den entsprechenden Infektionsumfeldern dominierten insgesamt 278 Ausbrüche mit 843 Fällen aus dem Setting des privaten Haushalts, der aber viele andere, nicht konkret definierbare Infektionsmöglichkeiten mit umfasst (- Tab. 3B). Alten- und Pflegeheime wa- 


\section{Leitthema}

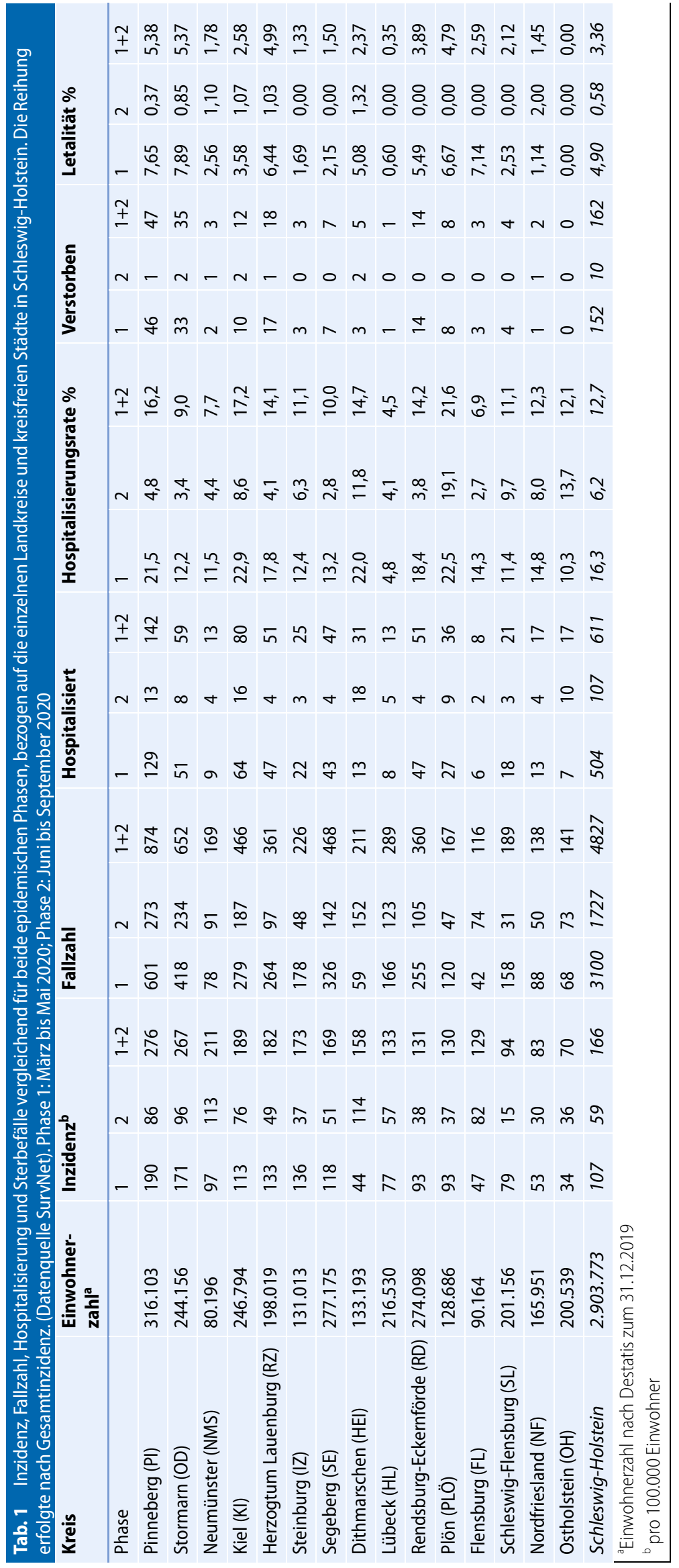


Tab. 2 Geschlecht, Alter, Klinik, Symptomatik, Risikokonstellation, Betreuung bzw. Tätigkeit nach $\S \S 23,33,36,42$ IfSG ${ }^{b}$ und Expositionsland vergleichend für die beiden epidemischen Phasen, bezogen auf die Patientengruppen (insgesamt, asymptomatisch, symptomatisch, hospitalisiert, verstorben). Die hospitalisierten und verstorbenen Patienten sind bei der Gruppe der Hospitalisierten ausgenommen, ebenso die Verstorbenen bei den Hospitalisierten. Phase 1: März bis Mai 2020; Phase 2: Juni bis September 2020. (Datenquelle SurvNet), MW: Mittelwert

\begin{tabular}{|c|c|c|c|c|c|c|c|c|c|c|c|c|c|c|c|}
\hline \multirow[b]{2}{*}{ Phase } & \multicolumn{3}{|c|}{ Insgesamt } & \multicolumn{3}{|c|}{ Asymptomatisch } & \multicolumn{3}{|c|}{ Symptomatisch } & \multicolumn{3}{|c|}{ Hospitalisiert } & \multicolumn{3}{|c|}{ Verstorben } \\
\hline & 1 & 2 & $1+2$ & 1 & 2 & $1+2$ & 1 & 2 & $1+2$ & 1 & 2 & $1+2$ & 1 & 2 & $1+2$ \\
\hline \multicolumn{16}{|l|}{ Geschlecht und Alter } \\
\hline Männlich, Anzahl & 1462 & 874 & 2336 & 248 & 333 & 581 & 956 & 479 & 1435 & 206 & 50 & 256 & 93 & 4 & 97 \\
\hline Männlich, MW Alter & 49,6 & 34,4 & 43,9 & 49,8 & 32,9 & 40,1 & 44,9 & 34,1 & 41,3 & 63,7 & 56,1 & 62,2 & 76,9 & 69,3 & 76,6 \\
\hline Weiblich, Anzahl & 1638 & 853 & 2491 & 286 & 321 & 607 & 1147 & 476 & 1623 & 186 & 49 & 235 & 59 & 6 & 65 \\
\hline Weiblich, MW Alter & 51,7 & 34,2 & 45,7 & 60,9 & 32,6 & 46,0 & 46,0 & 34,4 & 42,6 & 68,2 & 51,8 & 64,7 & 82,8 & 87,2 & 83,2 \\
\hline Gesamt, Anzahl & 3100 & 1727 & 4827 & 534 & 654 & 1188 & 2103 & 955 & 3058 & 392 & 99 & 491 & 152 & 10 & 162 \\
\hline Gesamt, MW Alter & 50,7 & 34,3 & 44,8 & 55,8 & 32,8 & 43,1 & 45,5 & 34,2 & 42,0 & 65,8 & 54,0 & 63,4 & 79,2 & 80,0 & 79,2 \\
\hline \multicolumn{16}{|l|}{ Altersgruppen } \\
\hline $0-9$ & 50 & 150 & 200 & 7 & 91 & 98 & 40 & 49 & 89 & 3 & 5 & 8 & 0 & 0 & 0 \\
\hline $10-19$ & 124 & 264 & 388 & 20 & 125 & 145 & 98 & 124 & 222 & 6 & 3 & 9 & 0 & 0 & 0 \\
\hline $20-29$ & 438 & 408 & 846 & 67 & 124 & 191 & 355 & 266 & 621 & 17 & 9 & 26 & 0 & 0 & 0 \\
\hline $30-39$ & 402 & 276 & 678 & 55 & 90 & 145 & 332 & 173 & 505 & 14 & 13 & 27 & 0 & 0 & 0 \\
\hline $40-49$ & 402 & 242 & 644 & 74 & 78 & 152 & 301 & 144 & 445 & 23 & 16 & 39 & 3 & 0 & 3 \\
\hline $50-59$ & 689 & 195 & 884 & 76 & 62 & 138 & 555 & 122 & 677 & 61 & 10 & 71 & 6 & 0 & 6 \\
\hline $60-69$ & 343 & 84 & 427 & 50 & 33 & 83 & 218 & 46 & 264 & 73 & 8 & 81 & 16 & 2 & 18 \\
\hline 70-79 & 293 & 51 & 344 & 67 & 21 & 88 & 114 & 18 & 132 & 93 & 14 & 107 & 42 & 3 & 45 \\
\hline $80-89$ & 279 & 39 & 318 & 82 & 21 & 103 & 75 & 9 & 84 & 89 & 17 & 106 & 60 & 3 & 63 \\
\hline 90-99 & 72 & 13 & 85 & 31 & 9 & 40 & 14 & 1 & 15 & 12 & 4 & 16 & 23 & 1 & 24 \\
\hline $100-119$ & 8 & 2 & 10 & 5 & 0 & 5 & 1 & 1 & 2 & 1 & 0 & 1 & 2 & 1 & 3 \\
\hline
\end{tabular}

\section{Klinik}

Klinik vorhanden

Ja, relevant für COVID-19

Ja, aber nicht relevant

Klinik nicht vorhanden

Nicht erhoben

Symptomatik

Fieber

Husten

Allgemeinsymptome

Schnupfen

Halsschmerzen

Dyspnoe

Durchfall

Geschmacksveränderung ${ }^{a}$

Geruchssinnveränderung ${ }^{\mathrm{a}}$

Pneumonie

Beatmung

Akutes Lungenversagen

(ARDS)

Tachykardie

Tachypnoe

Risikokonstellation

Herz-Kreislauf-Erkrankung

Nervensystemerkrankung

Lungenerkrankungen

Diabetes

Krebserkrankungen $\begin{array}{lllllllllllllllll}604 & 554 & 1158 & 0 & 0 & 0 & 499 & 511 & 1010 & 79 & 28 & 107 & 23 & 3 & 26\end{array}$

$\begin{array}{llllllllllllllll}142 & 219 & 361 & 142 & 219 & 361 & 0 & 0 & 0 & 29 & 20 & 49 & 5 & 3 & 8\end{array}$

$\begin{array}{llllllllllllllll}392 & 435 & 827 & 392 & 435 & 827 & 0 & 0 & 0 & 38 & 20 & 58 & 15 & 1 & 16\end{array}$

$\begin{array}{llllllllllllllll}3 & 39 & 42 & 0 & 0 & 0 & 0 & 0 & 0 & 1 & 2 & 3 & 0 & 0 & 0\end{array}$

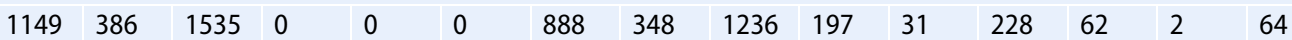

\begin{tabular}{lllllll|l|l|l|l|l|l|l|l|}
1477 & 464 & 1941 & 1 & 1 & 2 & 1248 & 425 & 1673 & 185 & 27 & 212 & 40 & 3 & 43
\end{tabular}

$\begin{array}{lllllllllllllllll}1272 & 584 & 1857 & 2 & 1 & 3 & 1040 & 532 & 1572 & 165 & 39 & 204 & 65 & 3 & 68\end{array}$

$\begin{array}{lllllllllllllllll}693 & 361 & 1054 & 0 & 1 & 1 & 647 & 346 & 993 & 39 & 8 & 47 & 6 & 0 & 6\end{array}$

$\begin{array}{llllllllllllllll}549 & 390 & 939 & 0 & 1 & 1 & 506 & 365 & 871 & 36 & 18 & 54 & 6 & 1 & 7\end{array}$

$\begin{array}{llllllllllllllll}266 & 36 & 302 & 0 & 0 & 0 & 137 & 20 & 157 & 83 & 11 & 94 & 46 & 3 & 49\end{array}$

$\begin{array}{llllllllllllllll}200 & 65 & 265 & 1 & 1 & 2 & 162 & 56 & 218 & 32 & 8 & 40 & 6 & 0 & 6\end{array}$

$\begin{array}{llllllllllllllll}123 & 223 & 346 & 0 & 0 & 0 & 115 & 207 & 322 & 8 & 13 & 21 & 0 & 0 & 0\end{array}$

$\begin{array}{lllllllllllllllll}105 & 181 & 286 & 0 & 0 & 0 & 97 & 170 & 267 & 8 & 9 & 17 & 0 & 0 & 0\end{array}$

$\begin{array}{lllllllllllllllll}64 & 9 & 73 & 0 & 0 & 0 & 1 & 1 & 2 & 30 & 5 & 35 & 33 & 3 & 36\end{array}$

$\begin{array}{lllllllllllllllll}31 & 4 & 35 & 0 & 0 & 0 & 2 & 2 & 4 & 10 & 0 & 10 & 19 & 2 & 21\end{array}$

$\begin{array}{llllllllllllllll}29 & 9 & 38 & 0 & 0 & 0 & 1 & 6 & 7 & 12 & 2 & 14 & 16 & 1 & 17\end{array}$

$\begin{array}{llllllllllllllll}9 & 6 & 15 & 0 & 0 & 0 & 4 & 4 & 8 & 3 & 2 & 5 & 2 & 0 & 2\end{array}$

$\begin{array}{lllllllllllllllll}7 & 3 & 10 & 0 & 0 & 0 & 2 & 1 & 3 & 2 & 2 & 4 & 3 & 0 & 3\end{array}$

\begin{tabular}{|c|c|c|c|c|c|c|c|c|c|c|c|c|c|c|}
\hline 442 & 123 & 565 & 19 & 11 & 30 & 204 & 58 & 262 & 132 & 21 & 153 & 57 & 5 & 62 \\
\hline 186 & 19 & 205 & 35 & 15 & 50 & 73 & 4 & 77 & 61 & 5 & 66 & 33 & 2 & 35 \\
\hline 149 & 56 & 205 & 18 & 18 & 36 & 81 & 32 & 113 & 44 & 6 & 50 & 14 & 2 & 16 \\
\hline 120 & 48 & 168 & 23 & 19 & 42 & 46 & 23 & 69 & 44 & 7 & 51 & 17 & 2 & 19 \\
\hline 106 & 24 & 130 & 23 & 11 & 34 & 44 & 10 & 54 & 37 & 7 & 44 & 17 & 0 & 17 \\
\hline
\end{tabular}




\section{Leitthema}

Tab. 2 (Fortsetzung)

\begin{tabular}{|c|c|c|c|c|c|c|c|c|c|c|c|c|c|c|c|}
\hline \multirow[b]{2}{*}{ Phase } & \multicolumn{3}{|c|}{ Insgesamt } & \multicolumn{3}{|c|}{ Asymptomatisch } & \multicolumn{3}{|c|}{ Symptomatisch } & \multicolumn{3}{|c|}{ Hospitalisiert } & \multicolumn{3}{|c|}{ Verstorben } \\
\hline & 1 & 2 & $1+2$ & 1 & 2 & $1+2$ & 1 & 2 & $1+2$ & 1 & 2 & $1+2$ & 1 & 2 & $1+2$ \\
\hline Nierenerkrankungen & 84 & 18 & 102 & 32 & 11 & 34 & 11 & 5 & 16 & 43 & 7 & 50 & 18 & 1 & 19 \\
\hline Immunschwäche & 69 & 13 & 82 & 7 & 5 & 12 & 35 & 5 & 40 & 20 & 4 & 24 & 12 & 0 & 12 \\
\hline Lebererkrankungen & 23 & 8 & 31 & 2 & 4 & 6 & 11 & 4 & 15 & 6 & 2 & 8 & 5 & 0 & 5 \\
\hline Schwangerschaft & 10 & 11 & 21 & 1 & 2 & 3 & 8 & 8 & 16 & 1 & 1 & 2 & 0 & 0 & 0 \\
\hline Postpartalphase & 3 & 2 & 5 & 0 & 1 & 1 & 2 & 1 & 3 & 1 & 0 & 1 & 0 & 0 & 0 \\
\hline \multicolumn{16}{|l|}{ Status nach $\S \S 23,33,36,42^{b}$} \\
\hline Betreut nach $\S 23$ & 77 & 30 & 107 & 23 & 22 & 45 & 13 & 1 & 14 & 51 & 12 & 0 & 11 & 3 & 14 \\
\hline Betreut nach $\S 33$ & 54 & 196 & 250 & 15 & 108 & 123 & 36 & 83 & 119 & 4 & 4 & 8 & 0 & 0 & 0 \\
\hline Betreut nach $\S 36$ & 303 & 37 & 340 & 127 & 28 & 155 & 76 & 7 & 83 & 51 & 2 & 53 & 68 & 2 & 70 \\
\hline Tätig nach § 23 & 346 & 78 & 424 & 43 & 25 & 68 & 279 & 53 & 332 & 21 & 0 & 21 & 3 & 0 & 3 \\
\hline Tätig nach $\S 33$ & 54 & 47 & 101 & 3 & 7 & 10 & 48 & 38 & 86 & 3 & 1 & 4 & 0 & 0 & 0 \\
\hline Tätig nach § 36 & 171 & 36 & 207 & 25 & 13 & 38 & 139 & 22 & 161 & 6 & 1 & 7 & 1 & 0 & 1 \\
\hline Tätig nach § 42 & 183 & 32 & 215 & 108 & 15 & 123 & 71 & 15 & 86 & 5 & 2 & 7 & 0 & 0 & 0 \\
\hline \multicolumn{16}{|l|}{ Expositionsländer } \\
\hline Schleswig-Holstein (SH) & 2027 & 909 & 1468 & 348 & 270 & 618 & 1305 & 555 & 1860 & 254 & 60 & 314 & 118 & 7 & 125 \\
\hline Hamburg $(\mathrm{HH})$ & 155 & 55 & 210 & 8 & 10 & 18 & 114 & 44 & 158 & 27 & 0 & 27 & 6 & 0 & 6 \\
\hline Deutschland, ohne SH und HH & 111 & 52 & 163 & 5 & 10 & 15 & 85 & 36 & 121 & 18 & 5 & 23 & 3 & 0 & 3 \\
\hline Österreich & 243 & 12 & 255 & 15 & 3 & 18 & 215 & 9 & 224 & 12 & 0 & 12 & 1 & 0 & 1 \\
\hline Mitteleuropa, weitere & 9 & 14 & 23 & 1 & 2 & 3 & 6 & 12 & 18 & 1 & 0 & 1 & 1 & 0 & 1 \\
\hline Nordeuropa & 10 & 14 & 24 & 2 & 0 & 2 & 7 & 14 & 21 & 0 & 0 & 0 & 1 & 0 & 1 \\
\hline Westeuropa & 29 & 17 & 46 & 3 & 8 & 11 & 23 & 9 & 32 & 3 & 0 & 3 & 0 & 0 & 0 \\
\hline Südeuropa & 62 & 70 & 132 & 5 & 34 & 39 & 52 & 35 & 87 & 3 & 1 & 4 & 2 & 0 & 2 \\
\hline Osteuropa & 4 & 40 & 44 & 0 & 11 & 11 & 3 & 28 & 31 & 1 & 1 & 2 & 0 & 0 & 0 \\
\hline Südosteuropa & 1 & 244 & 245 & 1 & 155 & 156 & 0 & 77 & 77 & 0 & 1 & 1 & 0 & 0 & 0 \\
\hline Europa, weitere & 3 & 0 & 3 & 0 & 0 & 0 & 3 & 0 & 3 & 0 & 0 & 0 & 0 & 0 & 0 \\
\hline Türkei & 4 & 93 & 97 & 0 & 50 & 50 & 3 & 38 & 41 & 1 & 0 & 1 & 0 & 1 & 1 \\
\hline Asien, weitere & 13 & 16 & 29 & 3 & 10 & 13 & 8 & 3 & 11 & 2 & 3 & 5 & 0 & 0 & 0 \\
\hline Amerika & 30 & 2 & 32 & 0 & 1 & 1 & 27 & 1 & 28 & 3 & 0 & 3 & 0 & 0 & 0 \\
\hline Afrika & 21 & 8 & 29 & 1 & 6 & 7 & 17 & 1 & 18 & 1 & 1 & 2 & 2 & 0 & 2 \\
\hline Australien & 4 & 0 & 4 & 0 & 0 & 0 & 2 & 0 & 2 & 2 & 0 & 2 & 0 & 0 & 0 \\
\hline Ausland, undifferenziert & 4 & 0 & 4 & 0 & 0 & 0 & 4 & 0 & 4 & 0 & 0 & 0 & 0 & 0 & 0 \\
\hline Keine Angabe & 370 & 181 & 551 & 55 & 27 & 82 & 229 & 93 & 322 & 64 & 21 & 85 & 18 & 2 & 20 \\
\hline
\end{tabular}




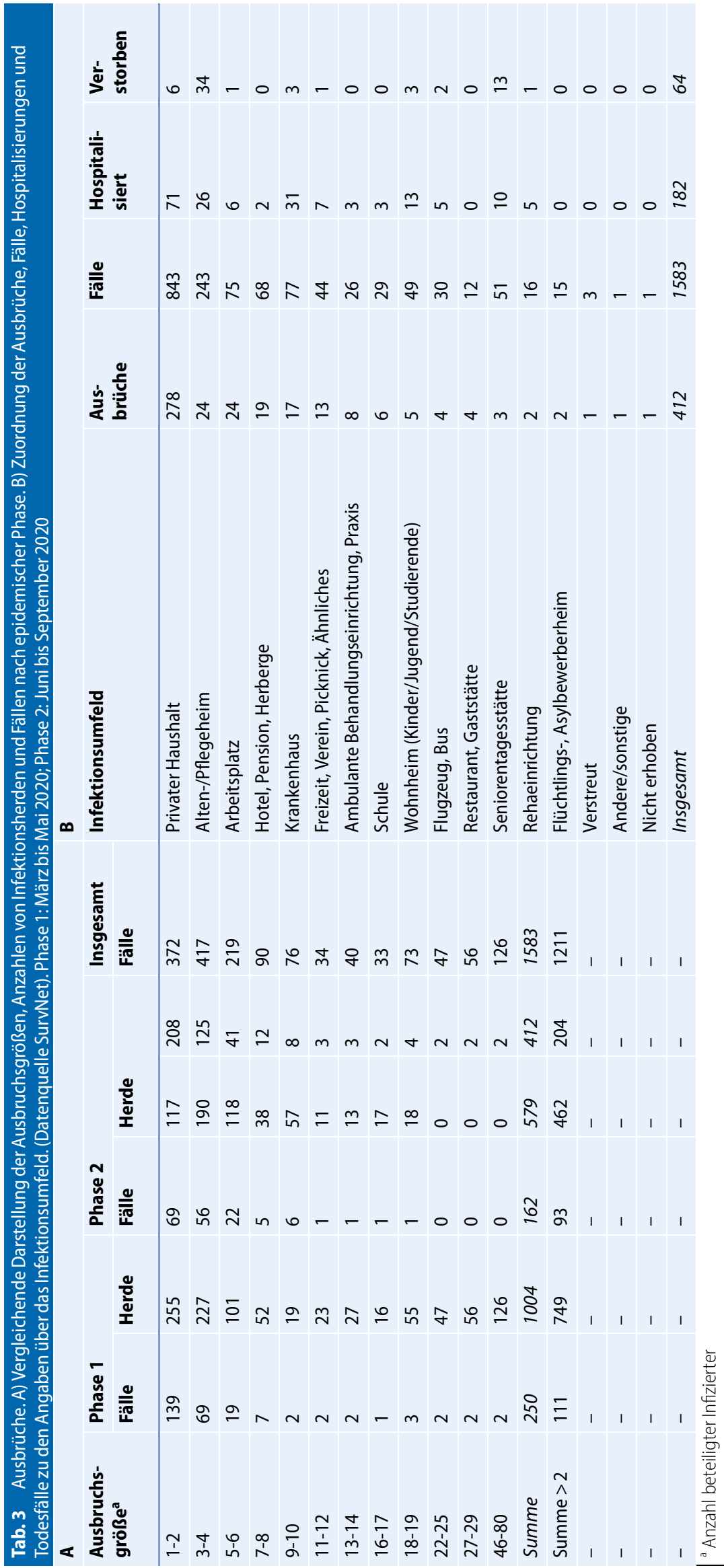

ren mit 24 Ausbrüchen und 243 Fällen bei 34 Verstorbenen hinsichtlich der Ausbruchs- und Fallzahl das zweitwichtigste Setting. 17 übergeordnete Ausbrüche betrafen mehrere Kreise und kreisfreie Städte. Der größte, kreisübergreifende Ausbruch in einem Schlachthof umfasste insgesamt 139 Personen. Außerdem sind unter diesen übergeordneten Ausbrüchen auch ein besonders großer Herd in einem Alten- und Pflegeheim mit 81 infizierten Personen und ein Ausbruch nach einem Spiel der Handballbundesliga enthalten.

Die Auswirkung der COVID-19-Pandemie auf die Gesamtmortalität wurde außerdem vergleichend für SchleswigHolstein und das gesamte Deutschland auf der Basis der veröffentlichten Daten des Statistischen Bundesamts (Destatis) untersucht. Die verfügbaren Daten des Jahres 2020 wurden ausgewertet und dem Mittelwert der Jahre 2016 bis 2019 gegenübergestellt (nicht dargestellt). Für Deutschland lag ein deutlicher Effekt der Pandemie auf die Gesamtmortalität für die 14.-17. Kalenderwoche vor (30.03.-26.04.2020). Dagegen war für Schleswig-Holstein ein sehr schwacher Effekt nur für die 16. Kalenderwoche nachzuweisen (13.04.-19.04.2020). Im Rahmen der einschränkenden Maßnahmen lösten sich diese Auffälligkeiten jedoch rasch auf. Auch wenn in Schleswig-Holstein eine beträchtliche Letalität der SARS-CoV-2-Infektion beobachtet wurde, wirkte sich diese noch nicht wesentlich auf die Gesamtmortalität aus. Damit kann weitestgehend ausgeschlossen werden, dass im Untersuchungszeitraum ein breiter, unerkannter Eintrag in die Allgemeinbevölkerung vorlag.

\section{Fazit}

Die Coronaviruspandemie verlief in Schleswig-Holstein im ersten Halbjahreszeitraum (März bis September 2020) im Vergleich zum Bundesgebiet relativ günstig mit einer der niedrigsten Landesinzidenzen. Für die Auswertung wurden die Meldedaten der ersten Phase von März bis Mai mit der zweiten Phase von Juni bis September verglichen. Die wesentlichen Unterschiede zwischen den epidemischen Phasen bestanden 
hinsichtlich der Hospitalisierung und Letalität, der Altersverteilung und der Expositionsländer. In der ersten Phase von März bis Mai 2020 dominierten schwere Erkrankungen älterer Personen mit hohen Raten der Hospitalisierung und Sterblichkeit. In der zweiten Phase von Juni bis September 2020 dominierten jüngere Personen mit niedrigeren Hospitalisierungs- und Sterberaten und mit deutlicher Reiseaktivität. Die meisten Ausbrüche wurden für private Haushalte dokumentiert. Die Pandemie hatte im Beobachtungszeitraum kaum Auswirkungen auf die Gesamtmortalität in Schleswig-Holstein.

\section{Korrespondenzadresse}

\section{Prof. Dr. Helmut Fickenscher}

Institut für Infektionsmedizin, Christian-

Albrechts-Universität zu Kiel und Universitäts-

klinikum Schleswig-Holstein

Brunswiker Str. 4, 24105 Kiel, Deutschland

fickenscher@infmed.uni-kiel.de

Danksagung. Großer Dank gilt allen meldenden Ärztinnen und Ärzten des Landes, den Mitarbeiterinnen und Mitarbeitern im Infektionsschutz des Öffentlichen Gesundheitsdienstes in den kommunalen Gesundheitsämtern und im Landes-Gesundheitsministerium (ganz besonders Frau Dr. Anne Marcic), den Mitarbeiterinnen und Mitarbeitern des RKI und den Landesmeldestellen anderer Bundesländer für ihre Unterstützung.

Funding. Open Access funding enabled and organized by Projekt DEAL.

\section{Einhaltung ethischer Richtlinien}

Interessenkonflikt. R. Rose, D. Scherer, G. Maschkowitz, C. Läubrich und H. Fickenscher geben an, dass kein Interessenkonflikt besteht.

Für dieses Manuskript wurden nur pseudonymisierte Meldedaten ausgewertet, die nach den Vorgaben des Infektionsschutzgesetzes durch die Gesundheitsämter erhoben wurden und im elektronischen Meldesystem SurvNet zur Verfügung standen. Da die Meldedaten nur durch das jeweils zuständige Gesundheitsamt und nicht durch die Landesmeldestelle decodiert werden können, haben die verwendeten Daten anonymisierten Charakter. Diese Software wird in einem geschützten Bereich eines Großrechners des Rechenzentrums der Christian-Albrechts-Universität zu Kiel betrieben.

Open Access. Dieser Artikel wird unter der Creative Commons Namensnennung 4.0 International Lizenz veröffentlicht, welche die Nutzung, Vervielfältigung, Bearbeitung, Verbreitung und Wiedergabe in jeglichem Medium und Format erlaubt, sofern Sie den/die ursprünglichen Autor(en) und die Quelle ordnungsge- mäß nennen, einen Link zur Creative Commons Lizenz beifügen und angeben, ob Änderungen vorgenommen wurden.

Die in diesem Artikel enthaltenen Bilder und sonstiges Drittmaterial unterliegen ebenfalls der genannten Creative Commons Lizenz, sofern sich aus der Abbildungslegende nichts anderes ergibt. Sofern das betreffende Material nicht unter der genannten Creative Commons Lizenz steht und die betreffende Handlung nicht nach gesetzlichen Vorschriften erlaubt ist, ist für die oben aufgeführten Weiterverwendungen des Materials die Einwilligung des jeweiligen Rechteinhabers einzuholen.

Weitere Details zur Lizenz entnehmen Sie bitte der Lizenzinformation auf http://creativecommons.org/ licenses/by/4.0/deed.de.

\section{Literatur}

1. Robert Koch-Institut (2000) Falldefinitionen für die Coronavirus-Krankheit-2019 (COVID-19) zur Übermittlung von Erkrankungs- oder Todesfällen und Nachweisen von Krankheitserregern, Stand vom 29.05.2020

2. Maschkowitz G, Rose R, Scherer D, Läubrich C, Fickenscher H (2020) Infektionsepidemiologischer Bericht über Meldepflichtige Krankheiten in Schleswig-Holstein für das Jahr 2020. Teil A: Coronavirus-Pandemie in Schleswig-Holstein im ersten Halbjahr 2020. Schmidt \& Klaunig, Kiel 